\title{
ARTIFICIAL SMALL PARAMETER METHOD-SOLVING MIXED BOUNDARY VALUE PROBLEMS
}

\author{
I. V. ANDRIANOV, J. AWREJCEWICZ, AND A. IVANKOV
}

Received 4 November 2004

The method of multiple scales is so popular that it is being rediscovered just about every 6 months.

A. H. Nayfeh [37, page 232]

A novel method for solving mixed boundary value problem is presented. A computational efficiency of the proposed method is illustrated using a few mechanical examples.

\section{Introduction}

The phrase quoted from Nayfeh's book applies also to the case of an artificial small parameter. Note that the introduction of the artificial small parameter is usually motivated either by a lack of a real physical small parameter or by a rather narrow application zone for the natural small parameter $[1,2,11,14,24]$. In general, the expression "small parameter" can be used in a different manner. Namely, the following key question occurs: is it possible to obtain a useful information directly through either a natural small parameter or an introduction of an artificial one (or through the application of a useful summation procedure)? This problem has been addressed in references [1, 12, 13, 26, 35].

In this respect it is worthwhile to speak rather directly on the "methods devoted to the development using a parameter" than to speak only on a "small parameter" [26].

From this point of view there is no difference between a real and an artificial small parameter. However, following tradition, the phrase "artificial small parameter" will be used. It is worth noticing that the idea of introducing a small parameter has been used in different branches of mathematics. For example, Dorodnitzyn [18] proposed the method of introduction of the parameter $\varepsilon$ into the input equations and the boundary conditions in the way that for $\varepsilon=0$ a simplified problem is obtained, whereas for $\varepsilon=1$ the original problem is described. In other words, Dorodnitzyn has applied the continuation method $[25,38,47,48]$ widely known in numerical mathematics. A serious problem occurred due to divergent series occurrence appearing for $\varepsilon=1$. In order to overcome the occurring difficulties, the so-called methods of analytical continuation have been proposed, but they did not work satisfactorily [33, 34, 40, 41, 42].

Some authors used the artificial parameter approach in a special way. Namely, they observed that a transition from $\varepsilon=0$ to $\varepsilon=1$ represents a homotopy transformation yielding today's accepted term the "homotopy perturbation technique" $[19,20,22,23$, $24,28,29,30,31]$. However, this mentioned technique can be satisfactorily applied only in connection with an effective method of summation. 
It has been already shown in references $[3,4,5,6,7,8,9,10,19,20]$ (see also $[1,11])$ that effective results are expected using the Padé approximations matched with homotopy perturbation techniques $[19,20]$.

This work is devoted to the description of the method presented in $[3,4,5,6,7,8,9,10$, $19,20]$ with an emphasis on its advantages. The paper is organized as follows. In Section 2 an introductory simple example is analyzed. Vibrations of clamped plate are discussed in detail in Section 3. In Section 4 the asymptotic method is applied to a static problem for a clamped plate. Here also the problem of solving an infinite system of algebraic equations is studied. Buckling of a rectangular plate is analyzed in Section 5. In Section 6 the results which have been obtained are summarized.

\section{Simple example}

In our first example a problem which has an exact solution, that is, frequencies of the clamped beam $(-0.5 l<x<0.5 l)$, is analyzed. Note that in a static case the Padé approach yields the exact solution directly. The equation being analyzed reads

$$
E I \frac{d^{4} w}{d X^{4}}-\omega^{2} \rho F w=0
$$

where $E$ is the Young modulus; $F, I$ are area and statics moment of the beam cross-section; $\rho$ is density; $\omega$ is frequency; $w$ is normal displacement; $X$ is a spatial coordinate.

The following nondimensional equation governs the vibrations of the beam:

$$
\frac{d^{4} w}{d x^{4}}-\lambda^{4} w=0
$$

where $x=X / l, \lambda^{4}=\omega^{2} \rho F l^{4} / E I$.

The boundary conditions have the following form:

$$
w=0, \quad \frac{d w}{d x}=0 \quad \text { for } x= \pm 0.5 .
$$

Let us introduce $\varepsilon$ in a way to get the following boundary conditions:

$$
w=0, \quad \varepsilon \frac{d w}{d x} \pm(1-\varepsilon) \frac{d^{2} w}{d x^{2}}=0 \quad \text { for } x= \pm 0.5
$$

Note that for $\varepsilon=0$ simple support is realized, whereas for $\varepsilon=1$ rigid clamping (2.3) occurs. If $0<\varepsilon<1$ elastic clamping appears.

A general solution to (2.2) reads

$$
w=C_{1} \operatorname{sh} \lambda x+C_{2} \operatorname{ch} \lambda x+C_{3} \sin \lambda x+C_{4} \cos \lambda x,
$$


and after satisfying the boundary conditions (2.4) it yields the following transcendental equations for both symmetric and antisymmetric vibrations with respect to the point $x=0$ :

$$
\begin{aligned}
& \varepsilon\left(\operatorname{ch} \frac{\lambda}{2} \sin \frac{\lambda}{2}+\operatorname{sh} \frac{\lambda}{2} \cos \frac{\lambda}{2}\right)+2(1-\varepsilon) \lambda \operatorname{ch} \frac{\lambda}{2} \cos \frac{\lambda}{2}=0 \\
& \varepsilon\left(\operatorname{ch} \frac{\lambda}{2} \sin \frac{\lambda}{2}+\operatorname{sh} \frac{\lambda}{2} \cos \frac{\lambda}{2}\right)+2(1-\varepsilon) \lambda \operatorname{sh} \frac{\lambda}{2} \sin \frac{\lambda}{2}=0
\end{aligned}
$$

Let us search for an eigenvalue $\lambda$ in the following series form:

$$
\lambda=\sum_{i=0}^{\infty} \lambda_{i} \varepsilon^{i}
$$

Substituting (2.8) into (2.6) (or (2.7)), and applying the classical splitting procedure with respect to the powers of $\varepsilon$, one gets

$$
\begin{aligned}
& \lambda=\pi n+\frac{\varepsilon}{\pi n}-\frac{\varepsilon^{2}}{2 \pi^{2} n^{2}}\left(\operatorname{th} \frac{\pi}{2} n-2 \pi n+\frac{2}{\pi n}\right)+\cdots, \quad n=1,3,5, \ldots, \\
& \lambda=\pi n+\frac{\varepsilon}{\pi n}-\frac{\varepsilon^{2}}{2 \pi^{2} n^{2}}\left(\operatorname{cth} \frac{\pi}{2} n-2 \pi n-\frac{2}{\pi n}\right)+\cdots, \quad n=2,4,6, \ldots
\end{aligned}
$$

Observe that since the series (2.9), (2.10) are divergent for $\varepsilon=1$, a summation procedure can be applied in order to receive a useful information. In our case a Padé approximation is applied $[12,13]$.

In what follows we are going to briefly describe the Padé approximation [12,13]. Let

$$
F(\varepsilon)=\sum_{i=0}^{\infty} c_{i} \varepsilon^{i}, \quad F[m, n](\varepsilon)=\sum_{i=0}^{m} a_{i} \varepsilon^{i}\left(\sum_{i=0}^{n} b_{i} \varepsilon^{i}\right)^{-1},
$$

where the coefficients $a_{i}$ and $b_{i}$ are defined through the following condition: the first $m+$ $n+1$ terms of the McLaurin series of $F[m, n](\varepsilon)$ coincide with the first $m+n+1$ terms of the $F(\varepsilon)$ series. Note that the rational function $F[m, n](\varepsilon)$ is called the $[m, n]$ th-order Pade approximation. The set of functions $F[m, n](\varepsilon)$ constitute the Padé table for different $m$ and $n$. The Padé approximation is unique for given $m$ and $n$ and creates a meromorphic continuation of the functions. Furthermore, in order to define the coefficients $a_{i}$ and $b_{i}$, knowing the series coefficients $c_{i}$, only a linear system of algebraic equations needs to be solved.

Padé transformation of the series part (2.9) (or (2.10)) has the form

$$
\lambda_{[1,1]}(\varepsilon)=\left(c_{0}+c_{1} \varepsilon\right)\left(d_{0}+d_{1} \varepsilon\right)^{-1}
$$

where $c_{0}=\lambda_{0}, d_{0}=1, c_{1}=\lambda_{1}+d_{1} \lambda_{0}, d_{1}=-\lambda_{2} / \lambda_{1}$.

We are now going to compare the eigenvalues of the problem (2.2), (2.3), and (2.4) yielded from the truncated series (2.9) (or (2.10)), and the Padé approximation (2.12) with a known exact value. A comparison is carried out only for the first eigenvalue, since the boundary conditions influence significantly only first eigenvalues. 
The first eigenvalue of a clamped beam is equal to $\lambda=4.712$, see [50]. In this case the series (2.9) with the first three terms (for $\varepsilon=1$ ) yields $\lambda=3.691$ (error amount of $27.68 \%$ ), whereas formula (2.12) gives $\lambda=4.429$ (error of $6.01 \%$ ).

Consider now the problem where a transition to transcendental equations can be omitted.

Beam vibrations are sought in the form

$$
w=\sum_{i=0}^{\infty} w_{i} \varepsilon^{i} .
$$

After a substitution of the series (2.13) into (2.2) and into the boundary conditions (2.4), and after a splitting with respect to $\varepsilon$, the following boundary value problems are obtained:

$$
\begin{aligned}
& \frac{d^{4} w_{0}}{d x^{4}}-\lambda_{0}^{4} w_{0}=0, \quad w_{0}=0, \quad \frac{d^{2} w_{0}}{d x^{2}}=0, \quad x= \pm 0.5, \\
& \frac{d^{4} w_{1}}{d x^{4}}-\lambda_{0}^{4} w_{1}-4 \lambda_{0}^{3} \lambda_{1} w_{0}=0, \quad w_{1}=0, \quad \frac{d w_{0}}{d x} \pm \frac{d^{2} w_{1}}{d x^{2}}=0, \quad x= \pm 0.5, \\
& \cos (n \pi x)+\frac{A}{\pi n}\left[\frac{(-1)^{0.5(n-1)}}{2 \operatorname{ch}(0.5 \pi n)} \operatorname{ch}(n \pi x)-x \sin (n \pi x)\right] \varepsilon \\
& +\frac{A}{2 \pi n}\left\{\frac{(-1)^{0.5(n+1)}}{2 \operatorname{ch}(0.5 \pi n)}\left[\left(\frac{1}{\pi n}+1\right) \operatorname{th}(0.5 \pi n)+\frac{2}{\pi^{2} n^{2}}-2\right] \operatorname{ch}(n \pi x)\right. \\
& +\left[\left(\frac{1}{\pi n}-\frac{1}{\pi^{2} n^{2}}+1\right) x \sin (n \pi x)+\frac{(-1)^{0.5(n-1)}}{2 \operatorname{ch}(0.5 \pi n)} x \operatorname{sh}(n \pi x)\right. \\
& \left.\left.-\frac{1}{\pi n} x^{2} \cos (n \pi x)\right]\right\} \varepsilon^{2}+\cdots, \quad n=1,3,5, \ldots, \\
& w=A \sin (n \pi x)+\frac{A}{\pi n}\left[\frac{(-1)^{0.5 n}}{2 \operatorname{sh}(0.5 \pi n)} \operatorname{sh}(n \pi x)-x \cos (n \pi x)\right] \varepsilon \\
& +\frac{A}{2 \pi n}\left\{\frac{(-1)^{0.5 n}}{2 \operatorname{sh}(0.5 \pi n)}\left[\left(\frac{1}{\pi n}+1\right) \operatorname{cth}(0.5 \pi n)+\frac{2}{\pi^{2} n^{2}}-2\right] \operatorname{sh}(n \pi x)\right. \\
& +\left[\left(\frac{1}{\pi n}+\frac{1}{\pi^{2} n^{2}}+1\right) x \cos (n \pi x)+\frac{(-1)^{0.5 n}}{\operatorname{sh}(0.5 \pi n)} x \operatorname{ch}(n \pi x)\right. \\
& \left.\left.-\frac{1}{\pi n} x^{2} \sin (n \pi x)\right]\right\} \varepsilon^{2}+\cdots, \quad n=2,4,6, \ldots
\end{aligned}
$$

Padé approximation of the series (2.16) (or (2.17)) gives

$$
w(\varepsilon)=\left(c_{0}+c_{1} \varepsilon\right)\left(d_{0}+d_{1} \varepsilon\right)^{-1}
$$

where $c_{0}=w_{0}, d_{0}=1, c_{1}=w_{1}+d_{1} w_{0}, d_{1}=-w_{2} / w_{1}$.

We compare the amplitude of the first harmonics of the clamped beam governed by (2.16) (or (2.17)) with the known exact value $w_{0}=1.133$, see [50].

Taking account of the three first terms of the series (2.16) for $\varepsilon=1$ gives $w_{0}=1.082$ (error $1.77 \%$ ). 
Observe that an exact bending moment with respect to the middle of the beam is $M(0)=-19.257$. Applying series (2.16), one gets $M(0)=-12.816$ (error $33.44 \%$ ), whereas with the Padé approximation (2.18) one gets $M(0)=-17.931$ (error $6.88 \%$ ).

Note that the exact value of a bending moment in the clamping area reads $M(0.5)=$ 31.405 , see [50]. Using part of the series (1.14) one gets $M(0.5)=23.086$, whereas applying the Padé approximation (1.16) one obtains $M(0.5)=28.671$ (error amount $8.70 \%$ ).

\section{Vibrations of a clamped plate}

We are going to analyze the free vibrations of a clamped rectangular plate. The governing equation reads

$$
D\left(\frac{\partial^{2}}{\partial x^{2}}+\frac{\partial^{2}}{\partial y^{2}}\right)^{2} w-\omega \rho_{1} h w=0
$$

where $D=E h^{3} /\left(12(1-\partial)^{2}\right), h$ is plate thickness; $\partial$ is Poisson coefficient; $\rho_{1}$ is density; $x$, $y$ are coordinates, $|x| \leq 0.5 a,|y| \leq 0.5 b$.

The input equation can be transformed to the following equivalent form:

$$
\nabla^{4} w-\lambda w=0
$$

where $\nabla^{4}=\left(\partial^{2} / \partial x^{2}+\partial^{2} / \partial y^{2}\right)^{2} ; \lambda=\omega^{2} \rho_{1} h b^{4} / D ; x=X / b ; y=Y / b$.

The following boundary conditions are applied:

$$
\begin{array}{lll}
w=0, & \frac{\partial w}{\partial x}=0 & \text { for } x= \pm 0.5 k \\
w=0, & \frac{\partial w}{\partial y}=0 & \text { for } y= \pm 0.5
\end{array}
$$

where $k=a / b$.

Observe that the parameter $\varepsilon$ is introduced to modify the boundary conditions (3.3) in the following way:

$$
\begin{aligned}
& w=0, \quad \varepsilon \frac{\partial w}{\partial x} \pm(1-\varepsilon) k \frac{\partial^{2} w}{\partial x^{2}}=0, \quad \text { for } x= \pm 0.5 k, \\
& w=0, \quad \varepsilon \frac{\partial w}{\partial y} \pm(1-\varepsilon) \frac{\partial^{2} w}{\partial y^{2}}=0, \quad \text { for } y= \pm 0.5 \text {. }
\end{aligned}
$$

For $\varepsilon=0$, simply supported boundary conditions are realized, whereas for $\varepsilon=1$, the contour of plate is clamped. Finally, for $0<\varepsilon<1$, an elastic clamping of the plate edges is realized.

A natural vibration frequency and an oscillation mode are sought in the following series forms:

$$
\lambda=\sum_{i=0}^{\infty} \lambda_{i} \varepsilon^{i}, \quad w=\sum_{i=0}^{\infty} w_{i} \varepsilon^{i} .
$$


Substituting the series (3.5) into (3.2), (3.4), and applying splitting with respect to $\varepsilon$, the following boundary conditions are obtained:

$$
\begin{gathered}
\nabla^{4} w_{0}-\lambda_{0} w_{0}=0, \\
w_{0}=0, \quad \frac{\partial^{2} w_{0}}{\partial x^{2}}=0, \quad \text { for } x= \pm 0.5 k, \\
w_{0}=0, \quad \frac{\partial^{2} w_{0}}{\partial y^{2}}=0, \quad \text { for } y= \pm 0.5, \\
\nabla^{4} w_{1}-\lambda_{1} w_{0}-\lambda_{0} w_{1}=0, \\
w_{1}=0, \quad \frac{\partial w_{0}}{\partial x^{2}} \pm k \frac{\partial^{2} w_{1}}{\partial x^{2}}=0, \quad \text { for } x= \pm 0.5 k, \\
w_{1}=0, \quad \frac{\partial w_{0}}{\partial y^{2}} \pm \frac{\partial^{2} w_{1}}{\partial y^{2}}=0, \quad \text { for } y= \pm 0.5 .
\end{gathered}
$$

Applying a selfadjoint property [37], (3.7), (3.10) yield eigenvalues of the problems (3.2), (3.4).

As a result, the following perturbation series is obtained:

$$
\begin{aligned}
& \lambda=\pi^{4}\left(n^{2} \eta_{n}+\frac{m^{2}}{k^{2}} \eta_{m}\right)+\frac{4 \pi^{2}}{k^{3}}\left(m^{2} \eta_{m}+k^{3} n^{2} \eta_{n}\right) \varepsilon \\
& +4 \frac{\left(m^{2} \eta_{m}+k^{2} n^{2} \eta_{n}\right)}{\left(k n \xi_{n}+m \xi_{m}\right)^{3}}\left\{2 \pi^{2}\left(n^{2} \eta_{n}+\frac{m^{2}}{k^{2}} \eta_{m}\right)\left(n \xi_{n}+m \xi_{m}\right)-4 \frac{\pi}{k^{3}}\left(m^{2} \eta_{m}+k^{3} n^{2} \eta_{n}\right)\right. \\
& \times\left[\frac{k^{2}\left(n \psi_{n}+m \psi_{m}\right)}{2 \pi\left(m^{2} \eta_{m}+k^{2} n^{2} \eta_{n}\right)}-\frac{1}{8 \pi}\left(\frac{k}{m \xi_{m}}+\frac{1}{n \xi_{m}}\right)\right] \\
& -\frac{m}{k^{3}}\left[\frac{\pi k \gamma_{m, n}}{2} \mathrm{th}^{(-1)^{m+1}}\left(\frac{\pi k \gamma_{m, n}}{2}\right)-1\right] \\
& -n\left[\frac{\pi \beta_{m, n}}{2} \mathrm{th}^{(-1)^{n+1}}\left(\frac{\pi \beta_{m, n}}{2}\right)-1\right]+\frac{k n^{2}}{m \xi_{n+1}} \\
& \left.+\frac{m^{2} \eta_{n}}{2 n k^{3}}+m n \frac{\left((m / k) \psi_{n} \xi_{m}+n \psi_{m} \xi_{n}\right)}{k^{2} n^{2} \eta_{n}+m^{2} \eta_{m}}\right\} \varepsilon^{2}+\cdots,
\end{aligned}
$$

where $\eta_{i}=2.5+1.5(-1)^{i}, \xi_{i}=-0.5+1.5(-1)^{i+1}$,

$$
\begin{gathered}
\gamma_{m, n}=\left(2 n \eta_{n}+\frac{m^{2}}{k^{2}} \eta_{m}\right)^{1 / 2}, \quad \beta_{m, n}=\left(n^{2} \eta_{n}+2 \frac{m^{2}}{k^{2}} \eta_{m}\right)^{1 / 2}, \\
\psi_{i}= \begin{cases}(-1)^{0.5(i-1)}, & i=1,3,5, \ldots, \\
1, & i=2,4,6, \ldots\end{cases}
\end{gathered}
$$

Note that the Padé transformation of the series (3.12) has the form

$$
\lambda(\varepsilon)=\left(c_{0}+c_{1} \varepsilon\right)\left(d_{0}+d_{1} \varepsilon\right)^{-1},
$$

where $c_{0}=\lambda_{0}, d_{0}=1, c_{1}=\lambda_{1}+d_{1} \lambda_{0}, d_{2}=-\lambda_{2} / \lambda_{1}$. 
The numerical simulation [50] yields $\lambda=5.998$ for the squared plate, the series part (3.12) gives $\lambda=4.854$ for $\varepsilon=1$ (error is $19.08 \%$ ), whereas formula (3.14) yields $\lambda=5.742$ (the error obtained is of $4.22 \%$ ).

\section{Static problem}

Consider a stress-strain state of a squared plate clamped along its contour $(-0.5 \leq x \leq$ $0.5,-0.5 \leq y \leq 0.5)$.

The plate bending equation reads

$$
\nabla^{4} w=q
$$

where $q=Q b^{4} D^{-1}$ and $Q$ denotes normal load.

The following boundary conditions are applied:

$$
\begin{array}{ll}
w=\frac{\partial w}{\partial x}=0 & \text { for } x= \pm 0.5 \\
w=\frac{\partial w}{\partial y}=0 & \text { for } y= \pm 0.5
\end{array}
$$

The perturbed boundary conditions (4.2) read

$$
\begin{array}{lll}
w=0, & \frac{\varepsilon \partial w}{\partial x} \pm \frac{(1-\varepsilon) \partial^{2} w}{\partial x^{2}}=0 & \text { for } x= \pm 0.5 \\
w=0, & \frac{\varepsilon \partial w}{\partial y} \pm \frac{(1-\varepsilon) \partial^{2} w}{\partial y^{2}}=0 & \text { for } y= \pm 0.5
\end{array}
$$

An equation governing the behavior of the bended plate is sought in the form

$$
\begin{aligned}
w= & \frac{4 q}{\pi^{5}} \sum_{i=1,3,5, \ldots}^{\infty} \frac{(-1)^{0.5(m-1)}}{m^{5}} \cos (\pi m x)\left[1-\frac{\alpha_{m} \operatorname{th} \alpha_{m}+2}{2 \operatorname{ch} \alpha_{m}} \operatorname{ch}(\pi m x)+\frac{1}{2 \operatorname{ch} \alpha_{m}} \pi m y \operatorname{sh}(\pi m y)\right] \\
& +\frac{1}{\pi^{2}} \sum_{i=1,3,5, \ldots}^{\infty} E_{m} \frac{(-1)^{0.5(m-1)}}{m^{2} \operatorname{ch} \alpha_{m}} \cos (\pi m x)\left[\pi m y \operatorname{sh}(\pi m y)-\alpha_{m} \operatorname{th} \alpha_{m} \operatorname{ch}(\pi m y)\right],
\end{aligned}
$$

where $\alpha_{m}=\pi m / 2, E_{m}$ are unknown coefficients.

The boundary conditions (4.4) yield the following system of linear algebraic equations:

$$
E_{i}+\varepsilon A_{i} E_{i}+\varepsilon \gamma_{i} \sum_{m=1,3,5, \ldots} \beta_{i m} E_{m}=\varepsilon B_{i}, \quad i=1,3,5, \ldots,
$$

where

$$
\begin{gathered}
A_{i}=\frac{1}{2 \pi i}\left(\operatorname{th} \alpha_{i}+\frac{\alpha_{i}}{\operatorname{ch}^{2} \alpha_{i}}\right)-1, \quad \alpha_{i}=0.5 \pi i, \quad \gamma_{i}=\frac{4 i}{\pi^{2}} \\
\beta_{i m}=\left(1+\frac{i^{2}}{m^{2}}\right)^{-2}, \quad B_{i}=\frac{2 q}{\pi^{4} i^{4}}\left(\frac{\alpha_{i}}{\operatorname{ch}^{2} \alpha_{i}}-\operatorname{th}^{2} \alpha_{i}\right)
\end{gathered}
$$


Applying

$$
E_{i}=\sum_{n=1}^{\infty} E_{i}^{(n)} \varepsilon^{n}, \quad i=1,3,5, \ldots
$$

and substituting (4.9) into (4.7), one gets

$$
E_{i}^{(1)}=B_{i}, \quad E_{i}^{(n+1)}=A_{i} E_{i}^{(n)}-\gamma_{i} \sum_{m=1,3,5, \ldots} \beta_{i m} E_{m}^{(n)}, \quad i=1,3,5, \ldots
$$

Note that the Pade transformation of the perturbation series (4.9) for $E_{i}$ has the form

$$
E_{i}=\frac{c_{0}+c_{1} \varepsilon+c_{2} \varepsilon^{2}}{d_{0}+d_{1} \varepsilon+d_{2} \varepsilon^{2}} \varepsilon
$$

where $c_{0}=E_{i}^{(1)}, d_{0}=1, c_{1}=E_{i}^{(2)}+d_{1} E_{i}^{(1)}, c_{2}=E_{i}^{(3)}+d_{1} E_{i}^{(2)}+d_{2} E_{i}^{(1)}$,

$$
\begin{aligned}
& d_{1}=\left(E_{i}^{(2)} E_{i}^{(5)}-E_{i}^{(3)} E_{i}^{(4)}\right)\left[E_{i}^{(2)} E_{i}^{(4)}-\left(E_{i}^{(3)}\right)^{2}\right]^{-1}, \\
& d_{2}=\left[\left(E_{i}^{(2)}\right)^{2}-E_{i}^{(3)} E_{i}^{(5)}\right]\left[E_{i}^{(2)} E_{i}^{(4)}-\left(E_{i}^{(3)}\right)^{2}\right]^{-1} .
\end{aligned}
$$

A deflection of the plate center computed through formula (4.6) with the application of $E_{i}$ represented by the five terms of the series (4.9) gives $1.797 \cdot 10^{-3} q$ for $\varepsilon=1$ (error of $42.60 \%$ ). A deflection computed with the use of $E_{i}$ through the Pade transformation (4.11) yields $1.275 \cdot 10^{-3} q$ (error of $1.20 \%$ ). A deflection obtained numerically yields $1.260 \cdot 10^{-3} q$, see [49].

The moment acting on the plate edges center reads

$$
M_{x( \pm 0.5,0)}=M_{y(0, \pm 0.5)}=\sum_{i=1,3,5, \ldots}^{\infty}(-1)^{0.5(i-1)} E_{i} \cos (\pi i x) .
$$

The moment value obtained numerically is equal to $-5.130 \cdot 10^{-2} q$. An application of the coefficients $E_{i}$ in the form of the perturbation series (4.9) gives the moment value $-3.888 \cdot 10^{-2} q$ (error of $24.20 \%$ ). Applying the coefficients $E_{i}$ obtained through Padé transformation yields $-5.173 \cdot 10^{-2} q$ (error of $0.83 \%$ ). The moment in the plate center is found through the formula $M_{x(0,0)}=M_{y(0,0)}$. The numerical solution [49] for $\nu=0.3$ yields $2.310 \cdot 10^{-2} q$. On the other hand, the moment values computed through application of the coefficients $E_{i}$ from the series (4.9) give $2.941 \cdot 10^{-2} q$ (error is of $27.32 \%$ ). Applying the coefficients $E_{i}$ obtained via Padé transformation, one gets $2.500 \cdot 10^{-2} q$ (error of $6.02 \%$ ).

The solution obtained emphasizes an important role of the introduction of an artificial small parameter while solving an infinite system of linear algebraic equations. Observe that the often used classical truncation method does not allow us to obtain a solution structure for complex mixed boundary value problems [36]. Although one may expect 
estimations of the solution coefficient asymptotics, this belongs rather to a separate task (see [36]). A method of introduction of the artificial small parameter allows us to account often for all system coefficients $x$. It plays an alternative role to the method of reduction and can be applied in order to estimate its accuracy.

It is worth noticing that in a similar application (see $[17,32])$ the number of operations required to obtain a solution of the system of equations with a finite number of unknowns dramatically decreases.

Finally, note that the artificial method of perturbation is effective also in the case of solution to the system of nonlinear equations $[3,4,5]$.

\section{Stability of a rectangular plate with mixed boundary conditions}

Consider the plate shown in Figure 5.1. It is assumed that the boundary conditions in the plate plane secure a homogeneous prebuckling plate state.

The input equation governing plate stability reads

$$
D\left(\frac{\partial^{2}}{\partial X^{2}}+\frac{\partial^{2}}{\partial Y^{2}}\right)^{2} w+P \frac{\partial^{2} w}{\partial X^{2}}=0
$$

where $P$ is a compressed load. The equivalent nondimensional equation reads

$$
\nabla^{4} w+N w_{x x}=0,
$$

where $x=X / b, y=Y / b, N=P b^{2} / D$.

The boundary conditions have the form

$$
\begin{gathered}
w=0, \quad w_{x x}=0 \quad \text { for } x= \pm 0.5 k, \\
w=0, \quad w_{y y}=\bar{H}(x)\left(w_{y y} \mp w_{y}\right) \quad \text { for } y= \pm 0.5,
\end{gathered}
$$

where $\bar{H}(x)=H(x-\gamma k)+H(-x-\gamma k), H(x)$ is the Heaviside function, $k=a / b$.

We now introduce the parameter $\varepsilon$ according to the scheme used earlier, namely,

$$
\begin{gathered}
w=0, \quad w_{x x}=0 \quad \text { for } x= \pm 0.5 k \\
w=0, \quad w_{y y}=\bar{H}(x)\left(w_{y y} \mp w_{y}\right) \quad \text { for } y= \pm 0.5 .
\end{gathered}
$$

The critical force $N$ and defection $w$ read

$$
N=\sum_{i=0}^{\infty} N_{i} \varepsilon^{i}, \quad w=\sum_{i=0}^{\infty} w_{i} \varepsilon^{i} .
$$


Substituting (5.5) into (5.2), (5.4), the following series of boundary conditions is obtained:

$$
\begin{gathered}
\nabla^{4} w_{0}+N_{0} w_{0 x x}=0, \\
w_{0}=0, \quad w_{0 x x}=0 \quad \text { for } x= \pm 0.5 k, \\
w_{0}=0, \quad w_{0 y y}=0 \quad \text { for } y= \pm 0.5, \\
\nabla^{4} w_{j}+N_{0} w_{j x x}=-\sum_{i=0}^{j-1} N_{j-i} w_{i x x}, \\
w_{j}=0, \quad w_{j x x}=0 \quad \text { for } x= \pm 0.5 k, \\
w_{j}=0, \quad w_{j y y}=\mp \bar{H}(x) \sum_{i=0}^{j-1} w_{i y} \quad \text { for } y= \pm 0.5 .
\end{gathered}
$$

A solution to the boundary value problems (5.6), for $j=1,2,3$, yields

$$
\begin{aligned}
& N=\pi^{2} \frac{k^{2}}{m^{2}}\left(n^{2}+\frac{m^{2}}{k^{2}}\right)^{2}+4 k^{2} \frac{n^{2}}{m} \gamma_{m m} \varepsilon \\
& +\frac{k^{2}}{\pi^{2} m^{2}}\left\{4 \pi^{2} n^{2} \gamma_{m m}-\frac{2 n^{2}}{\left(n^{2}+m^{2} / k^{2}\right)} \gamma_{m m}(0.5 \alpha \text { th } 0.5 \alpha-1)\right. \\
& -4 \pi^{2} n^{2} \sum_{\substack{i=1,3,5, \ldots . \\
i \neq m}}^{\infty} \gamma_{i m}^{2}\left(\alpha_{i} \text { th } 0.5 \alpha_{i}-\left\{\begin{array}{c}
\beta_{i} \text { th } 0.5 \beta_{i} \\
-\gamma_{i} \operatorname{tg} 0.5 \gamma_{i}
\end{array}\right\}\right) \\
& \left.-\frac{n^{2} m}{\left(n^{2}+m^{2} / k^{2}\right)^{2}}\left(n^{2}-\frac{m^{2}}{k^{2}}\right) \gamma_{m m}^{2}\right\} \varepsilon^{2}+\cdots \\
& \left\{\begin{array}{l}
m(i-m)>n^{2} k^{2} \\
m(i-m)<n^{2} k^{2}
\end{array}\right\} \\
& \alpha=\pi \sqrt{2 \frac{m^{2}}{k^{2}}+n^{2}}, \quad \alpha_{i}=\pi \sqrt{i\left(\frac{i+m}{k^{2}}+\frac{n^{2}}{m}\right)}, \quad \beta_{i}=\pi \sqrt{i\left(\frac{i+m}{k^{2}}-\frac{n^{2}}{m}\right)}, \\
& \gamma_{i}=\pi \sqrt{i\left(\frac{n^{2}}{m}-\frac{i-m}{k^{2}}\right)} \text {. }
\end{aligned}
$$

Note that the Padé approximation for the series (5.7) has the form

$$
N(\varepsilon)=\frac{a_{0}+a_{1} \varepsilon}{b_{0}+b_{1} \varepsilon}
$$

where $a_{0}=N_{0}, a_{1}=N_{1}+b_{1} N_{0}, b_{0}=1, b_{1}=-N_{2} / N_{1}$.

In what follows we are going to estimate an error of the solution which is obtained in a limiting case corresponding to full clamping of the plate sides $y= \pm 0.5$. The exact solution gives (for the squared plate) $N=8.6044 \pi^{2}$ [15], for $\varepsilon=1$ formula (5.8) gives 


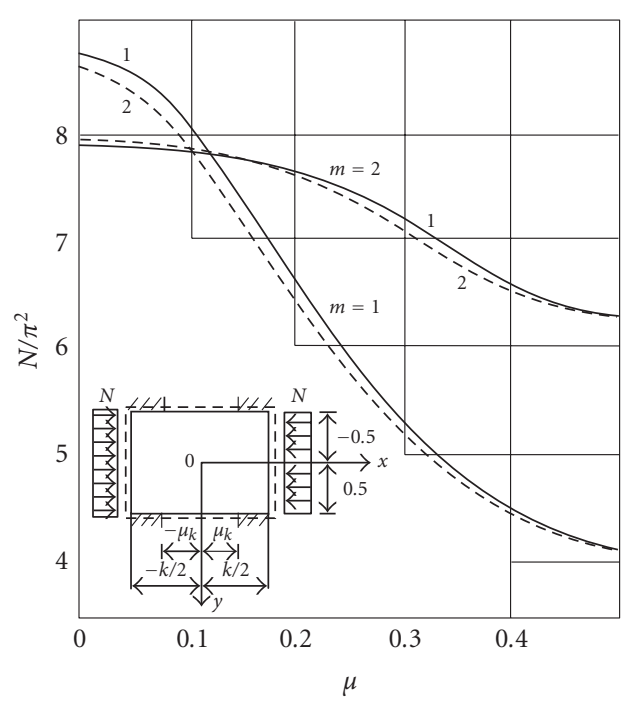

Figure 5.1. Comparison of the stability estimation results of the plate with mixed boundary conditions using conditions either from this paper or from those based on $R$-function application.

$N=8.7136 \pi^{2}$ (error of $1.27 \%$ ); the series (5.7) yields $N=4.7757 \pi^{2}$ (error of $44.5 \%$ ). Numerical solution of the transcendental equation for $m=2$ yields $N=7.6913 \pi^{2}$, see [15]; formula (5.8) for $\varepsilon=1$ gives $N=7.7156 \pi^{2}$ (error of $0.31 \%$ ), whereas the series (5.7) gives $N=6.4456 \pi^{2}$ (error of $16.13 \%$ ).

It is worth noticing that in the places where boundary conditions are changing rapidly one may expect singularities. Since these singularities are known $[43,44]$, they can be introduced into a solution using known methods for matching singular solutions and known asymptotic solutions [39].

The critical force $N$ versus geometrical ratios of the types of mixed boundary conditions $\mu$ is reported in Figure 5.1. The results obtained with the help of the described method and the $R$-function method [46] are represented by curves 1 and 2, respectively. In the given graphs one may distinguish two zones: the first one is for $\mu \in[0.0,0.15]$, where the plate buckling is associated with an occurrence of two half-waves in direction $x$; the second one is for $\mu \in[0.15,0.5]$, where the plate buckling loss is characterized through an occurrence of one half-wave in $x$-direction. Therefore, for $\mu=0.15$ one may expect buckling through either the first or second buckling form.

The described method allows for an investigation of the influence of clamping stiffness $\varepsilon$ on the buckling force $N$. The dependence of the buckling force $N$ versus the parameter $\varepsilon$ is shown in Figure 5.2 for some values of $\mu$. It should be emphasized that in the case of an elastic clamping of the edges $y= \pm 0.5$ with no mixed boundary conditions, the additional equilibrium forms appear for $\varepsilon=0.96$. Buckling with an occurrence of one (two) halfwave in the $x$-direction may appear for $\varepsilon<0.96(\varepsilon>0.96)$. A simultaneous occurrence of the first and second equilibrium forms, depending on the parameter $\varepsilon$, takes place for mixed boundary conditions. Furthermore, the additional equilibrium forms for $\mu \rightarrow 0$ 


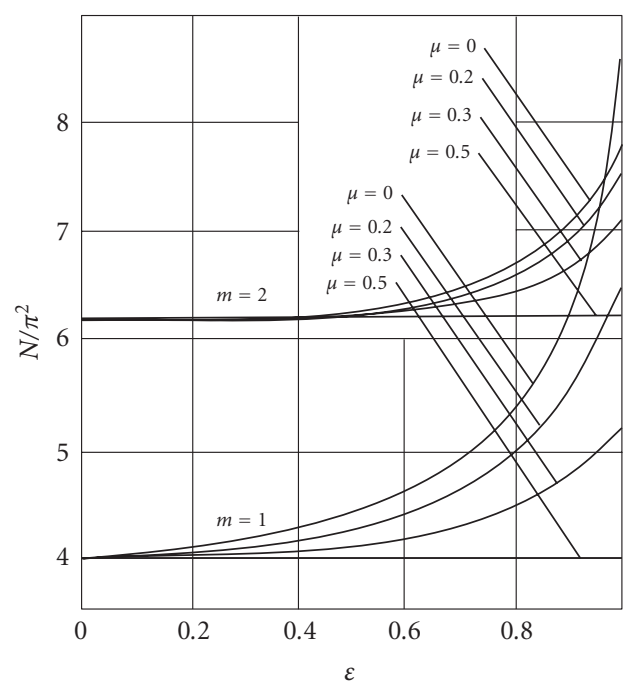

Figure 5.2. Investigation of the influence of clamping space dimension on buckling load of the plate.

appear for $\varepsilon \rightarrow 1.0$. A threshold value of $\mu=0.25$ corresponds to plate stability loss one buckled half-wave in $x$-direction for $\varepsilon=1$.

For the plate shown in Figure 5.3, the solutions (5.7), (5.8) still hold. However, in the latter case the coefficients $\gamma_{i m}$ should be sought in the form

$$
\gamma_{i m}= \begin{cases}2 \gamma-\frac{(-1)^{m}}{4 \pi m} \sin (2 \pi \gamma m) & \text { for } i=m, \\ \frac{4}{\pi\left(m^{2}-i^{2}\right)}[i \sin (\pi \gamma i) \cos (\pi \gamma m)-m \sin (\pi \gamma m) \cos (\pi \gamma i)] & \text { for } i \neq m .\end{cases}
$$

The dependence of $N$ versus $\mu$ is reported in Figure 5.3, whereas the dependence $N(\varepsilon)$ is shown in Figure 5.4 for the same values of $\mu$. Curve 1 corresponds to formulas (5.7), (5.8); curve 2 corresponds to the $R$-function method [46]; curve 3 (2.5) represents the results reported in [27] (see [21]), and the bullets represent the experimental data shown in [21]. To conclude, a good coincidence is achieved for both computational and experimental data for the whole variation of the parameter $\mu$.

\section{Concluding remarks}

The proposed computational method possesses advantages in comparison with the known methods devoted to solving the problems associated with mixed boundary conditions, that is, the methods of Bubnov-Galerkin et al. (see [36]). Namely, it does not require a priori knowledge of the shapes of deformed surfaces. Furthermore, the proposed approach does not lead also to a high-order system of transcendental equations, as in the case of dynamical edge effect method [16]. 


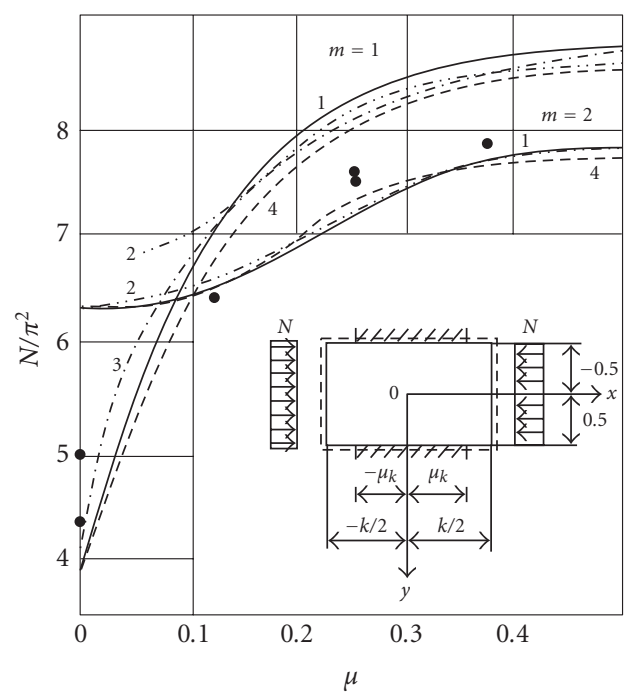

Figure 5.3. Comparison of the computational results of plate stability estimation with mixed boundary conditions obtained through our proposed method, $R$-function approach, and experimental results.

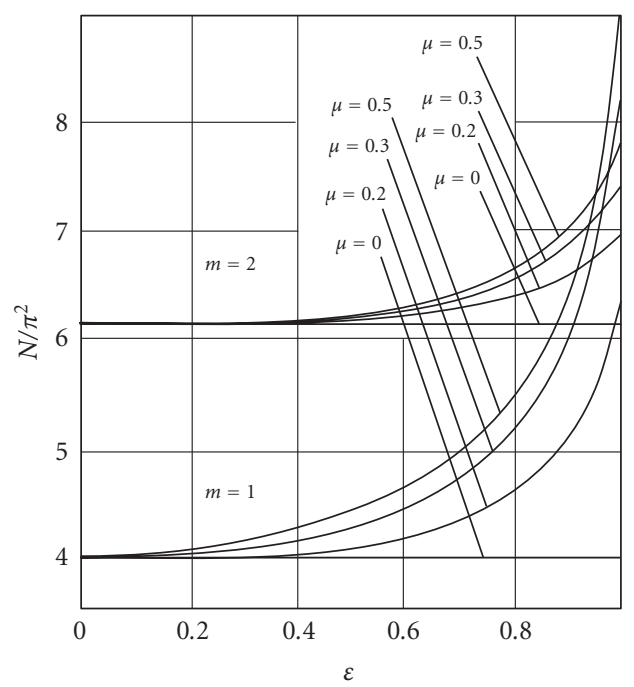

Figure 5.4. Investigation of the influence of clamping part length on buckling load of the plate.

The proposed asymptotic method allows for solution representation in an analytic form, which is important when applying any optimal design for solution of direct problems. Applying FEM or BEM for initial $\varepsilon$ and $\mu$, one may also solve a boundary value problem. 
It should be emphasized, however, that FEM method is universal with respect to a space fulfilled by a plate. It is rather difficult to apply an asymptotic method to complex form spaces, since they require knowledge of the analytical solution of zero-order approximation. Besides, applying an asymptotic method does not provide an easier way to introduce higher accuracy, since the construction of higher approximations is rather difficult. However, one may require a solution obtained through two methods in order to control the reliability of the obtained approximate solution. In the case of complex plate forms, the results obtained through the asymptotic method can serve either as the initial values for FEM or as tests for FEM, if a transition from a complex to simple geometry is possible.

The proposed approach can also be applied to solve 3D problems of elasticity, hydromechanics, and so forth. It allows for a significant extension of the classical method of variables separation and the Fourier method.

One may also match the asymptotic method with the Bubnov-Galerkin-type methods. Indeed, after obtaining an infinite set of linear algebraic equations [36], the method of artificial small parameter can be applied easily.

Finally, in the context of the approach which has been introduced and described, the domain decomposition method can be modified [45].

\section{References}

[1] I. V. Andrianov and J. Awrejcewicz, New trends in asymptotic approaches: summation and interpolation methods, Appl. Mech. Rev. 54 (2001), no. 1, 69-92.

[2] I. V. Andrianov, J. Awrejcewicz, and R. G. Barantsev, Asymptotic approaches in mechanics: new parameters and procedures, Appl. Mech. Rev. 56 (2003), no. 1, 87-110.

[3] I. V. Andrianov and V. V. Danishevs'kyy, Asymptotic investigation of the nonlinear dynamic boundary value problem for rod, Tech. Mech. 15 (1995), no. 1, 53-55.

[4] - Asymptotic approach for non-linear periodical vibrations of continuous structures, J. Sound Vibration 249 (2002), no. 3, 465-481.

[5] I. V. Andrianov, V. Z. Gristchak, and A. O. Ivankov, New asymptotic method for the natural, free and forced oscillations of rectangular plates with mixed boundary conditions, Tech. Mech. 14 (1994), no. 3-4, 185-193.

[6] I. V. Andrianov and A. O. Ivankov, Using Padé approximants in the method of introduction parameter for investigation of biharmonic equation with complicated boundary conditions, USSR Comp. Math. Math. Physics 27 (1987), no. 1, 193-196.

[7] Solution of mixed bending plate problem by modified boundary conditions perturbation method, Doklady AN Ukr. SSR A 1 (1988), 33-36 (Russian).

[8] - New asymptotic method for solving of mixed boundary value problem, Free Boundary Problems in Continuum Mechanics (Novosibirsk, 1991), Internat. Ser. Numer. Math., vol. 106, Birkhäuser, Basel, 1992, pp. 39-45.

[9] - On the solution of the plate bending mixed problems using modified technique of boundary conditions perturbation, ZAMM 73 (1993), no. 2, 120-122.

[10] I. V. Andrianov, E. G. Kholod, and V. A. Chernetsky, Asymptotic-based method for a plane elasticity mixed boundary eigenvalue problem, Journal Theoret. Appl. Mech. 32 (1994), no. 3, 701-709.

[11] J. Awrejcewicz, I. V. Andrianov, and L. I. Manevitch, Asymptotic Approaches in Nonlinear Dynamics: New Trends and Applications, Springer Series in Synergetics, Springer, Berlin, 1998. 
[12] G. A. Baker Jr., Essentials of Padé Approximants, Academic Press [A subsidiary of Harcourt Brace Jovanovich, Publishers], New York-London, 1975.

[13] G. A. Baker Jr. and P. Graves-Morris, Padé approximants, 2nd ed., Encyclopedia of Mathematics and Its Applications, vol. 59, Cambridge University Press, Cambridge, 1996.

[14] C. M. Bender, K. A. Milton, Moshe Moshe, S. S. Pinsky, and L. M. Simmons Jr., Logarithmic approximations to polynomial Lagrangeans, Phys. Rev. Lett. 58 (1987), no. 25, 2615-2618.

[15] F. Bloom and D. Coffin, Handbook of Thin Plate Buckling and Postbuckling, Chapman \& Hall/CRC, Florida, 2001.

[16] V. V. Bolotin, Random Vibrations of Elastic Systems, Monographs and Textbooks on Mechanics of Solids and Fluids: Mechanics of Elastic Stability, vol. 8, Martinus Nijhoff Publishers, Dordrecht, 1984.

[17] S. Cabay and B. Domzy, Systems of linear equations with dense univariate polynomial coefficients, J. Assoc. Comput. Mach. 34 (1987), no. 3, 646-660.

[18] A. A. Dorodnitzyn, Using of Small Parameter Method for Numerical Solution of Mathematical Physics Equations, Num. Meth. Cont. Mech. Probl. Solving, VZ AN SSSR, Moscow, 1969.

[19] R. El. Mokhtari, J. M. Cadou, and M. Potier-Ferry, A two grid algorithm based on perturbation and homotopy method, C. R. Mechanique 330 (2002), 825-830.

[20] A. Elhage-Hussein, M. Potier-Ferry, and N. Damil, A numerical continuation method based on Padé approximants, Internat. J. Solids Structures 37 (2000), no. 46-47, 6981-7001.

[21] M. Hamada, Y. Inoue, and H. Hasimoto, Buckling of simply supported partially clamped rectangular plates uniformly compressed in one direction, Bull. ISME 10 (1967), no. 37, 35-40.

[22] J.-H. He, Homotopy perturbation technique, Comput. Methods Appl. Mech. Engrg. 178 (1999), no. 3-4, 257-262.

[23] A coupling method of homotopy technique and a perturbation technique for non-linear problems, Int. J. Non-Linear Mech. 35 (2000), 37-43.

[24] - A review on some new recently developed nonlinear analytical techniques, Int. J. Nonlinear Sci. Numer. Simul. 1 (2000), no. 1, 51-70.

[25] M. Kawahara, N. Yoshimura, K. Nakagawa, and H. Ohsaka, Steady and unsteady finite element analysis of incompressible viscous fluid, Internat. J. Numer. Methods Engrg. 10 (1976), no. 2, 437-456.

[26] Ya. F. Kayuk, Some Problems of the Methods Splitting by Parameter, Naukova Dumka, Kiev, 1991.

[27] L. M. Kier and V. Stal, Eigenvalue problem for rectangular plate with mixed boundary values, J. Appl. Mech. Tech. Phys. 2 (1972), 196-204.

[28] S. J. Liao, A second-order approximate analytical solution of a simple pendulum by the process analysis method, J. Appl. Mech. Tech. Phys. (ASME) 59 (1992), no. 4, 970-975.

[29] __ An approximate solution technique not depending on small parameters: a special example, Int. J. Non-Linear Mech. 30 (1995), no. 3, 371-380.

[30] A uniformly valid analytic solution of two-dimensional viscous flow over a semi-infinite flat plate, J. Fluid Mech. 385 (1999), 101-128.

[31] S. J. Liao and A. T. Chwang, Application of homotopy analysis method in nonlinear oscillations, Trans. ASME J. Appl. Mech. 65 (1998), no. 4, 914-922.

[32] V. Meleshko, Selected topics in the history of the two-dimesional biharmonic problem, Appl. Mech. Rev. 56 (2003), no. 1, 33-85.

[33] N. A. Meller and A. A. Dorodnitsyn, Approaches to the solution of stationary Navier-Stokes equations, USSR Comp. Math. \& Math. Phys. 8 (1968), no. 2, 205-217.

[34] N. A. Meller and A. A. Dorodnitzyn, Application of the small parameter method to the solution of Navier-Stokes equations, Fluid Dynamics Trans. 5 (1971), no. 2, 67-82.

[35] T. Miloh (ed.), Mathematical Approaches in Hydrodynamics, Society for Industrial and Applied Mathematics (SIAM), Philadelphia, 1991. 
[36] R. T. Moenck and J. H. Carter, Approximate algorithms to derive exact solutions to systems of linear equations, Symbolic and Algebraic Computation (EUROSAM '79, Internat. Sympos., Marseille, 1979), Lecture Notes in Comput. Sci., vol. 72, Springer, Berlin, 1979, pp. 65-73.

[37] A. H. Nayfeh, Perturbation Methods, Wiley Classics Library, Wiley-Interscience [John Wiley \& Sons], New York, 2000.

[38] A. K. Noor and J. M. Peters, Tracing post-limit-point paths with reduced basis technique, Comput. Methods Appl. Mech. Engrg. 28 (1981), 217-240.

[39] I. F. Obraztsov, B. V. Nerubaŭlo, and I. V. Andrianov, Asymptotic Methods in the Structural Mechanics of Thin-Walled Structures, Mashinostroenie, Moscow, 1991.

[40] B. V. Pal'tsev, The expansion of solutions Dirichlet's problem and a mixed problem for a biharmonic equation in a series of solutions of reducing problems, USSR Comp. Math. \& Math. Phys. 6 (1966), no. 1, 59-72.

[41] Small-parameter method in the boundary value problem for an Oseen system, USSR Comp. Math. \& Math. Phys. 7 (1967), no. 5, 236-266.

[42] _ Convergence of expansions with respect to a small parameter introduced into the boundary conditions of the solutions of boundary value problem for Navier-Stokes equations, USSR Comp. Math. \& Math. Phys. 10 (1970), no. 2, 133-157.

[43] V. Z. Parton and P. I. Perlin, Mathematical Methods of the Theory of Elasticity. Vol. 1, Mir, Moscow, 1984.

[44] Mathematical Methods of the Theory of Elasticity. Vol. 2, Mir, Moscow, 1984.

[45] A. Quarteroni and A. Valli, Domain Decomposition Methods for Partial Differential Equations, Numerical Mathematics and Scientific Computation, The Clarendon Press, Oxford University Press, New York, 1999.

[46] V. L. Rvachëv and L. V. Kurpa, R-Functions in Problems of the Theory of Plates, Naukova Dumka, Kiev, 1987.

[47] V. E. Shamanskii, Methods of Numerical Solution of Boundary Value Problems by Electronic Computer, Naukova Dumka, Kiev, 1966.

[48] J. M. T. Thompson and A. C. Walker, The nonlinear perturbation analysis of discrete structural systems, Internat. J. Solids Structures 4 (1968), 757-768.

[49] S. P. Timoshenko and S. Woinowsky-Krieger, Theory of Plates and Shells, 2nd ed., McGraw-Hill, New York, 1987.

[50] W. Weaver Jr., S. P. Timoshenko, and D. M. Young, Vibration Problems in Engineering, Wiley, New York, 1990.

I. V. Andrianov: Institute of General Mechanics, RWTH Aachen, Templergraben 64, 52062 Aachen, Germany

E-mail address: igor_andrianov@hotmail.com

J. Awrejcewicz: Department of Automatics and Biomechanics, Technical University of Łódź, 1/15 Stefanowski Street, 90-924 Łódź, Poland

E-mail address: awrejcew@p.lodz.pl

A. Ivankov: Pridneprovsk State Academy of Civil Engineering and Architecture, 24a Chernishevskogo Street, 49005 Dnepropetrovsk, Ukraine

E-mail address: ali959@sstr.ru 


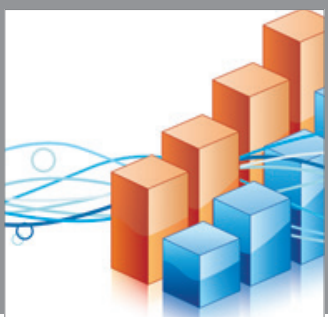

Advances in

Operations Research

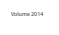

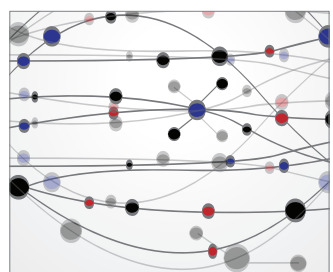

\section{The Scientific} World Journal
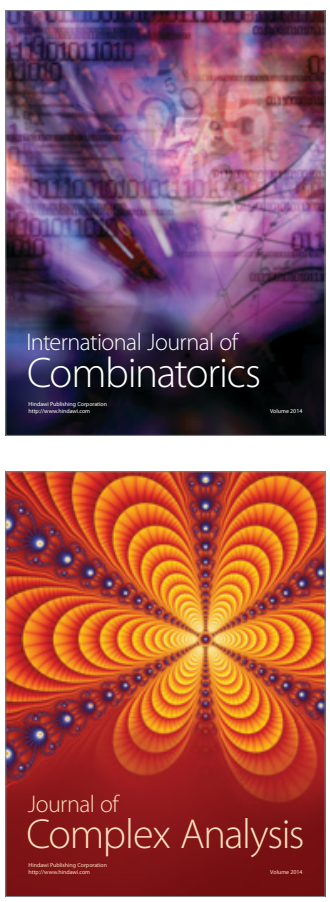

International Journal of

Mathematics and

Mathematical

Sciences
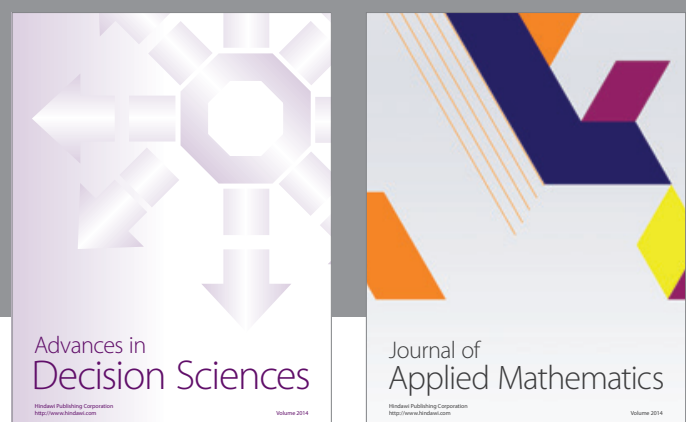

Journal of

Applied Mathematics
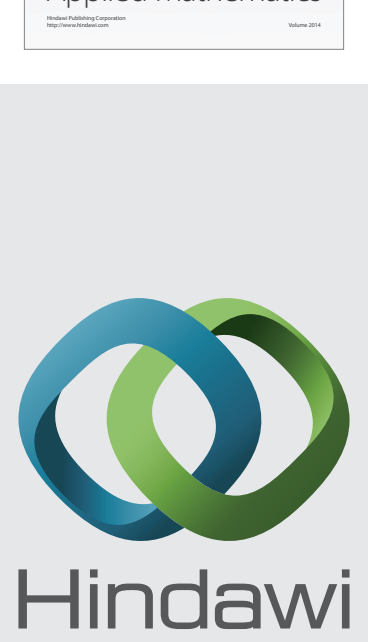

Submit your manuscripts at http://www.hindawi.com
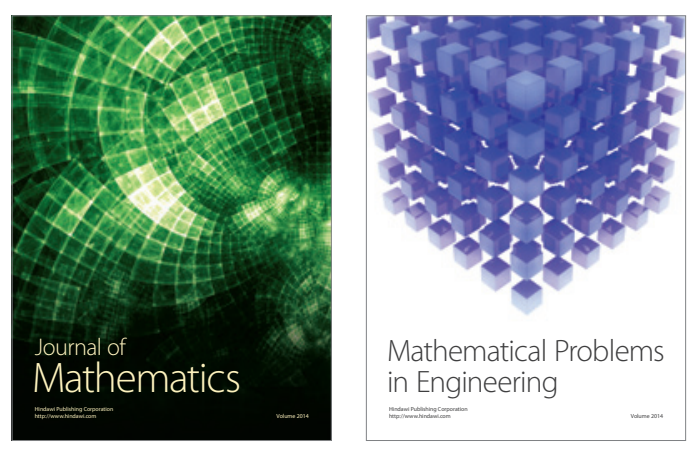

Mathematical Problems in Engineering
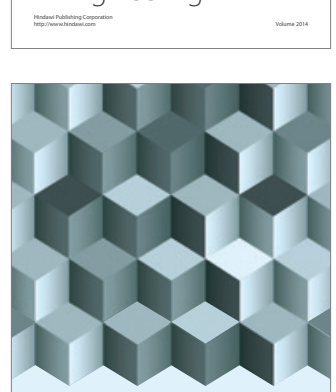

Journal of

Function Spaces
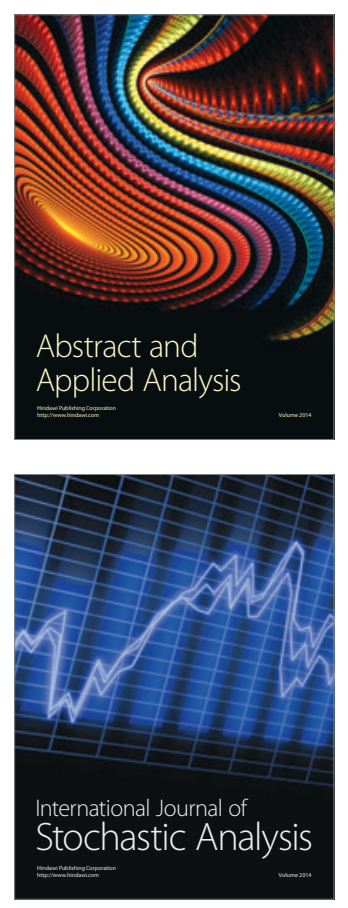

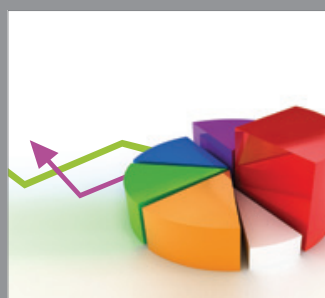

ournal of

Probability and Statistics

Promensencen
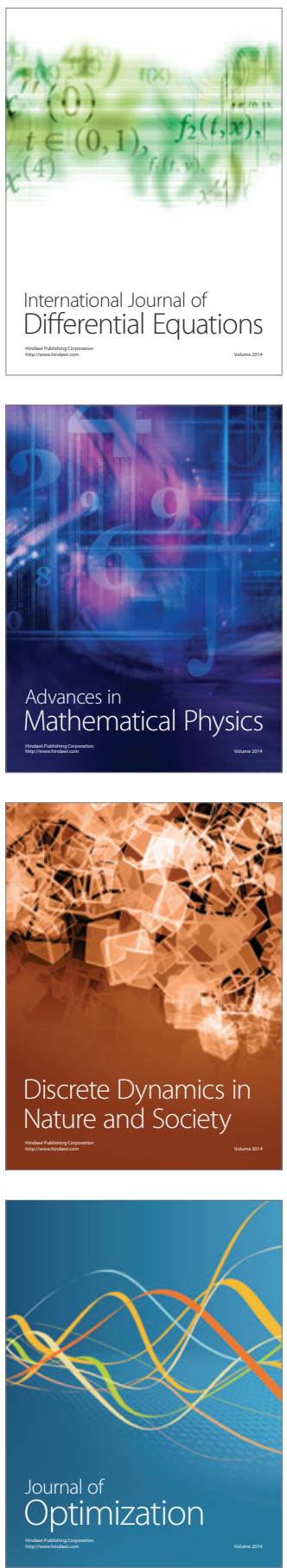